\title{
IMPLICATIONS OF ABUNDANCE MEASUREMENTS OF THE INTRACLUSTER MEDIUM
}

\author{
W. Forman, C. Jones and L. David \\ Smithsonian Astrophysical Observatory, Cambridge, MA, USA
}

\begin{abstract}
The correlation of $M_{\text {gas }} / M_{\text {stellar }}$ with the intracluster gas temperature in clusters of galaxies has implications for the efficiency of galaxy formation and predicts trends for the heavy element abundances in these systems.
\end{abstract}

\subsection{INTRODUCTION}

The discovery of a correlation between the ratio of the gas mass to stellar mass, $M_{g a s} / M_{s t e l l a r}$, and the gas temperature ( $T_{g a s}$, a measure of the depth of the potential) in groups and clusters of galaxies has important implications for some models of the formation of large scale structure in the Universe (David et al. 1989a). In this contribution we describe the importance of determining the heavy element (iron) abundances of groups and clusters with gas temperatures, that span the range of the correlation of $M_{\text {gas }} / M_{\text {stellar }}$ vs. $T_{g a s}$.

We will confine our discussion to the cold dark matter scenario for the formation of structure in the Universe which is described by Blumenthal et al. (1984). In this scenario, it is assumed that the baryonic mass in the Universe is just that component of the mass that is luminous. Thus, in groups and clusters of galaxies the predominant baryonic (luminous) components are the gas mass, $M_{\text {gas }}$, and the stellar mass (in galaxies), $M_{\text {stellar }}$. Thus, $M_{\text {luminous }}=M_{\text {gas }}+M_{\text {stellar }}$ and the remaining mass, $M_{\text {virial }}-M_{\text {luminous }}$, consists of cold, dark matter and is not baryonic. What can we learn from abundance determinations of the hot gas in groups and clusters in a cold, dark matter scenario?

\subsection{IMPLICATIONS OF THE CORRELATION OF $M_{\text {gas }} / M_{\text {stellar }}$ WITH $T_{\text {gas }}$}

As described by Jones et al. (1989; especially Figure 1), the ratio of the gas to stellar mass, $M_{\text {gas }} / M_{\text {stellar }}$, increases from unity in groups and poor clusters with low temperatures $(\sim 2 \mathrm{keV})$ to values of 3-6 in systems with high gas temperatures (6-10 $\mathrm{keV}$ ). This correlation, combined with an understanding of the production of heavy elements, predicts a correlation of heavy element abundance with gas temperature.

The groups which are luminous x-ray sources are dense systems and have stellar populations comparable to rich clusters (Morgan et al. 1975). Also, the correlation of galaxy population with local density (Dressler 1978, and Postman and Geller 
1984) supports the similarity of the galaxy populations in the groups and clusters. Therefore, the production of heavy elements should be directly proportional to the stellar light, or equivalently stellar mass, since comparable populations will have similar mass-to-light ratios. Thus, the larger the ratio of gas mass to stellar mass, the more dilute the stellar products like iron. Since $M_{\text {gas }} / M_{\text {stellar }}$ increases with increasing $T_{\text {gas }}$, we predict that hotter clusters (those with larger $M_{\text {gas }} / M_{\text {stellar }}$ ) will have lower iron abundances than cooler clusters. This prediction assumes that the clusters and groups are closed systems, i.e., no gas is expelled or accreted.

Figure 1 shows the quantitative prediction for the correlation of iron abundance with gas temperature. The two solid curves are derived by taking a simple parameterization for the dependence of $M_{g a s} / M_{\text {stellar }}$ on $T_{g a s}$ and assuming that enriched material is expelled from galaxies only during an early wind phase during which Type II supernovae can readily drive a galactic wind (see David et al. 1989b). The two curves assume different initial mass functions (the upper curve has a power law exponent $\alpha=2$ and the lower curve has $\alpha=2.5$ ). Note that an amount of enriched material equal to that expelled in the wind is produced by stellar evolution and could be liberated by ram pressure stripping. The present estimates of supernova

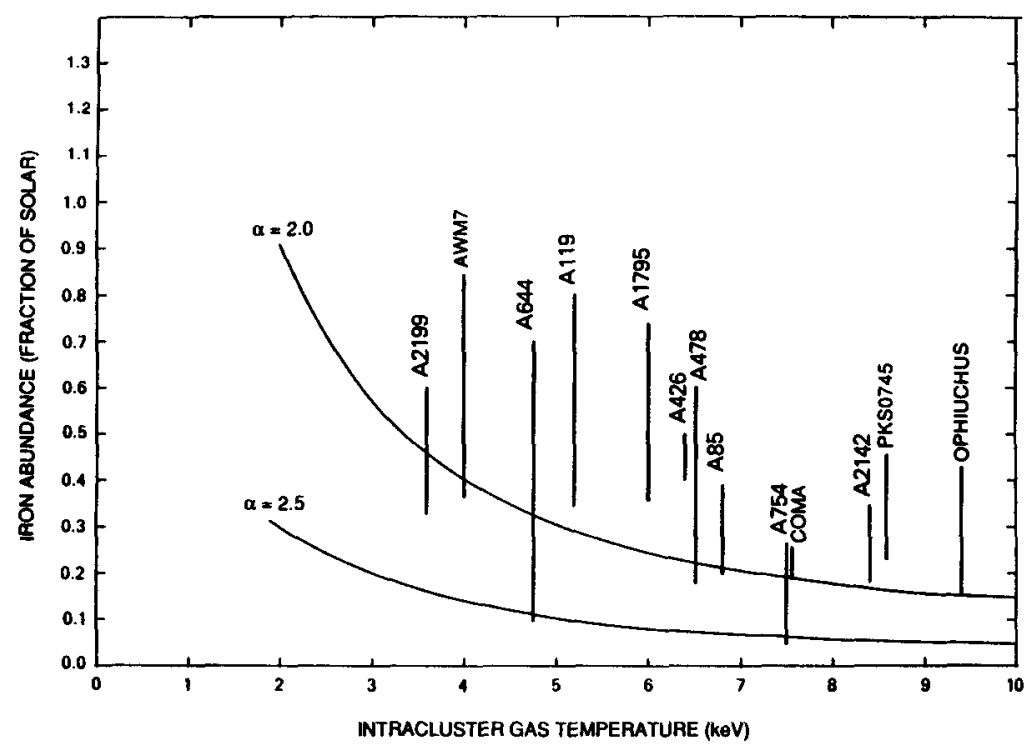

Figure 1. The iron abundance (as a fraction of the solar value) is plotted against gas temperature. The data are taken from Henriksen (1985), Hughes et al. (1988) and Arnaud et al. (1987). The smooth curves are predictions based on a parameterization of the relation between $M_{\text {gas }} / M_{\text {stellar }}$ and $T_{\text {gas }}$ as well as a model for the evolution of stars with two different initial mass functions. 
yields can explain the observed heavy element abundances in the intracluster gas as Figure 1 shows. The ejected gas is extremely enriched and is diluted to the observed values by mixing with the predominantly primordial component of the intracluster medium.

The previous discussion assumes that the groups and clusters are closed systems, i.e., gas is not expelled or accreted in significant quantities. This can be tested by observing clusters with progressively lower temperatures. If ejection becomes important below some temperature, $T_{\text {crit }}$, then one would observe an increasing heavy element abundance from the hottest clusters down to those with temperatures equal to $T_{\text {crit }}$. Below $T_{\text {crit }}$, the winds would serve to expel enriched material and the abundance would decline (or remain constant) as the gas temperature decreases further.

\subsection{IMPORTANCE OF FUTURE ABUNDANCE DETERMINATIONS}

Future experiments will provide greatly enhanced spectroscopic capabilities and will open new avenues for studies of the intracluster gas. The ability to obtain spatially resolved spectra is most important for studies of the intracluster gas since it provides a means to determine abundance and temperature gradients. Below we mention two important observations and their implications.

First, the measurement of temperature profiles in clusters allows the direct determination of the underlying gravitational mass (luminous and dark matter). Assuming hydrostatic equilibrium and spherical symmetry, the gravitating mass is given by

$$
M_{g r a v}(r) \propto\left(\frac{d \ln \rho}{d \ln r}+\frac{d \ln T_{g a s}}{d \ln r}\right) T_{g a s}(r) r
$$

where $\rho$ is the gas density and $r$ is the radial distance from the cluster center. Only M87 and Centaurus (NGC4696) have measured density and temperature gradients (Fabricant and Gorenstein 1983, Matilsky et al. 1985). For most clusters $T_{g a s}(r)$ remains unknown (or very uncertain). Thus, ASTRO-D, AXAF, and XMM can add immeasurably to our present knowledge.

Second, abundance measurements of clusters will have implications for a variety of problems. Average abundances, in the context of the previous discussion of the changing ratio of gas to stellar mass, can place constraints on models for galaxy formation (e.g., biasing) in some scenarios. Radial gradient determinations will provide information on the origin of the heavy elements. Since the galaxies and gas in clusters have different distributions, the radial variation of abundances could have implications for the existence of Population III objects. Finally, abundance 
measurements at different redshifts will tell us about the enrichment mechanisms of the intracluster medium and the evolution of the stellar populations producing the enriched material.

Acknowledgements

We would like to thank M. Fonseca and K. Modestino for their help in preparing this manuscript. This work was supported by the Smithsonian Institution Scholarly Studies Program and by NASA contract NASA-30751.

\section{REFERENCES}

Arnaud, K. A., Johnstone, R. M., Fabian, A. C., Crawford, C. S., Nulsen, P. E. J., Shafer, R. A., and Mushotzky, R. F. 1987, MNRAS, 227, 241.

Blumenthal, G. R., Faber, S. M., Primack, J. R., and Rees, M. J. 1984, Nature, $311,517$.

David, L., Arnaud, K., Forman, W., and Jones, C. 1989a, preprint.

David, L., Forman, W., and Jones, C. 1989b, this volume.

Dressler, A. 1978, Ap. J., 226, 55.

Fabricant, D. and Gorenstein, P. 1983, Ap. J., 267, 535.

Henriksen, M. 1985, Ph.D. Thesis (University of Maryland).

Hughes, J. P., Yamashita, K., Okumura, Y., Tsunemi, H., and Matsuoka, M. 1988, Ap. J., 327, 615.

Jones, C., David, L., Forman, W. 1989, this volume.

Matilsky, T., Jones, C., and Forman, W. 1985, Ap. J., 291, 621.

Morgan, W. W., Kayser, S., and White, R. A. 1975, Ap. J., 199, 545.

Postman, M. and Geller, M. 1984, Ap. J., 281, 95. 